\title{
Studies on the selective localization of multi-walled carbon nanotubes in blends of poly(vinylidene fluoride) and polycaprolactone
}

\author{
L. $L i^{1,2}$, W-H. Ruan ${ }^{2}$, M-Q. Zhang ${ }^{2}$, M-Z. Rong ${ }^{2}$ \\ ${ }^{1}$ Materials Science Institute, The School of Chemistry and Chemical Engineering, Zhongshan University, 510275 \\ Guangzhou, P. R. China \\ ${ }^{2}$ National Engineering Laboratory for Plastic Modification and Processing, National-certified Enterprise Technology \\ Center, Kingfa Science and Technology Co. LTD., 510275 Guangzhou, P. R. China.
}

Received 22 May 2014; accepted in revised form 17 August 2014

\begin{abstract}
A ternary system based on blends of poly(vinylidene fluoride)(PVDF) and polycaprolactone (PCL) and made the multi-walled carbon nanotubes (MWNT) selective distribution in PCL phases via melt blending has been studied. The studies on conductivity of MWNT/PCL/PVDF composites with different proportions of PVDF and PCL showed that the conductivity was good in the mass ratio range of PVDF/PCL from 70/30 to 40/60 and that of other matching was poor. The scanning electron microscope (SEM) observation of the composites revealed co-continuous morphologies of PVDF and PCL were formed in composites under suitable proportion of PVDF to PCL. The interfacial tensions of PVDF and PCL melts was tested at mixing temperature of $200^{\circ} \mathrm{C}$, the calculated wetting coefficient indicated that MWNT would selectively distribute in PCL phase owing to interfacial effects. Transmission electron microscope (TEM) observation further confirmed that co-continuous morphologies of PVDF and PCL could appear with the increase of PCL content, as indicated by MWNT selective localization in PCL.As the result, the desired structure of double percolation was built in MWNT/PCL/PVDF composites, which have much higher conductivity than that of PVDF or PCL composites with the same MWNT content. Through a strategy of selective localization of MWNT, PVDF based material with low percolation threshold $(<0.5 \mathrm{wt} \%)$ was acquired which held promise for potential use in large-scale industrial applications.
\end{abstract}

Keywords: polymer composites, poly(vinylidene fluoride), multi-walled carbon nanotubes, double percolation, low percolation threshold

\section{Introduction}

Conductive and dielectric materials based on PVDF have attracted considerable interests from the scientific community and industry, owing to their wide use in electrical and electronic industry [1-3]. The main advantages of PVDF-based composites are good mechanical flexibility and tunable properties for making different types of devices. Due to the insulating properties of PVDF, conductive fillers, especially nanoparticles such as CNT, carbon black, graphene and metal particles, need to be added to the matrix. CNT/PVDF composites are one of the most important types of conductive materials because of the excellent electric and mechanical properties of CNT [4-7]. Solution mixing CNT with PVDF, involving sonication, is usually preferred in the lab studies of CNT/PVDF composites and relatively low percolation threshold of CNT could be achieved. But solution mixing is a solvent-consuming process, which is not suitable for industrial production. CNT/PVDF nanocomposites prepared by melt compounding, which is a feasible way for large-scale pro-

\footnotetext{
${ }^{*}$ Corresponding author, e-mail: cesrwh@mail.sysu.edu.cn (C) BME-PT
} 
duction, have been reported with the manufacture of MWNT reaching industrial scale in recent years [8-10]. However the percolation thresholds are much higher $(>2.5 \mathrm{wt} \%)$ than those of solution mixing ones, indicating that the major problem of melt compounding CNT/PVDF composites is the poor dispersion of CNT in polymer matrices. Many studies were carried out to deal with the problem [11-14]. The design of double percolation structure in composites is one of the methods to reduce the percolation threshold of conductive fillers [15-21].The concept of double percolation was first proposed by Sumita et al. [21] in immiscible blends that were filled with carbon black. Two requirements are needed to realize the double percolation structure in composites. One requires co-continuous blend morphologies, the other needs selective filling of only one of the blend phases (double percolation). Preferential localization of conductive filler in one of the phases is commonly explained by the differences in interfacial energies of the filler and the corresponding polymer, which originates from the different polarities and surface energies. Some of the authors compared the predication of the wetting coefficient or equivalent equations that described the localization of fillers in blends to their experimental observations [22-24]. However, it was very difficult to get the interfacial tensions of polymer melt at the processing temperature. The interfacial tensions of polymer at room temperature were used to predict the wetting coefficient [22, 23, 25], but sometimes results deviating from actual situation might be obtained. There are a few reports about CNT/PVDF composites prepared by double percolation concept $[23,26]$. But the interfacial effect for predicting the localization of conductive fillers was not studied at all. To understand the interfacial effect on selective distribution of MWNT, the interfacial tensions of PVDF melt at processing temperature should be investigated.

In order to prepare PVDF based conductive material with low percolation threshold by melt blending, a ternary system of MWNT/PCL/PVDF for double percolation was designed in this paper. The pendant drop method $[27,28]$ was applied to measure the interfacial tensions of polymer melts at processing melt for selective localization of MWNT. Formation of co-continuous morphologies in composites by adjusting the proportions of PVDF to PCL were investigated. The study should contribute to acquir- ing a basic knowledge of the development of low electrically percolated MWNT/PVDF composites on industrial scale.

\section{Experimental section \\ 2.1. Materials}

The PVDF resin (solef1010) with an average molecular weight of 45000 was purchased from the Solvay Company (Brussels, Belgium). The multi-walled carbon nanotubes (MWNT) with a length of 10-30 $\mu \mathrm{m}$ and an outer diameter of $10-20 \mathrm{~nm}$ were obtained from the Chengdu Institute of Organic Chemistry (China). PCL resin with an average molecular weight of 80000 was purchased from the Esun Company (Shenzhen, China).

\subsection{Sample preparations}

The PVDF matrix and MWNT were dried in the vacuum oven at $90^{\circ} \mathrm{C}$ and $\mathrm{PCL}$ was dried at $50^{\circ} \mathrm{C}$ for $12 \mathrm{~h}$ before being mixed. The PCL and MWNT were first mixed in the Haake mixer at $110^{\circ} \mathrm{C}$ for $10 \mathrm{~min}$ and MWNT/PCL composites with different concentrations of MWNT were obtained. Then MWNT/PCL composites were added into PVDF according to pre-fixed proportions of PVDF to PCL. The MWNT/ PCL/PVDF blends were mixed in the Haake mixer at $200^{\circ} \mathrm{C}$ for $10 \mathrm{~min}$.

\subsection{Characterization}

Conductive properties of MWNT/PCL/PVDF composites were tested by a high-resistance meter (Agilent 4339B, United States of America). The granules of MWNT/PCL/PVDF blends were pressed with a fixed pressure in a hydraulic machine and the sheets of MWNT/PCL/PVDF composites were obtained. The sample size is $20 \mathrm{~mm} \times 20 \mathrm{~mm} \times 0.2 \mathrm{~mm}$ at least. The wide measurement range is $10^{3}$ to $1.6 \cdot 10^{16} \Omega$ with a $10 \mathrm{~ms}$ high-speed measurement.

The interfacial tensions of PCL and PVDF melt were tested by the Krüss DSA 100 (Krüss Company, Ltd., Germany) apparatus with the pendant drop method at $200^{\circ} \mathrm{C}$, respectively.

The phase structure of the PVDF and PCL was characterized by a scanning electron microscope (SEM, JEOL JSM-5400, Japan) with an accelerating voltage of $7.0 \mathrm{kV}$. The samples were held in liquid nitrogen for $40 \mathrm{~min}$ and then impact fractured for SEM analysis. The fractured surface was etched by acetic acid in order to dissolve the PCL phase. The samples were gold sputtered before SEM observation. The 
dispersion of MWNT in PVDF/PCL was observed with a transmission electron microscope (TEM, FEITecnai G2 Spirit) with an accelerating voltage of $120 \mathrm{kV}$. Ultrathin sections of the samples were prepared in liquid nitrogen before TEM observation.

\section{Results and discussion}

Conductive properties of the composites with different mass ratio of PVDF and PCL contained $1.5 \mathrm{wt} \%$ of MWNT were shown in Figure 1. It is clear to see that conductivity of the composites changed with the increase of PCL content. There was an abrupt increase in conductivity above PVDF/PCL of 90/10, a plateau from PVDF/PCL of 70/30 to 40/60, and a sharp decrease after PVDF/PCL of 40/60. The results indicated that the phase morphologies of the composites changed through adjusting the content of PCL, leading to the changes of the conductive properties.

SEM images of $1.5 \mathrm{wt} \%$ MWNT/PCL/PVDF composites were given in Figure 2. In order to distinguish PCL phase from PVDF phase, the samples were etched by acetic acid to remove PCL in the cross-section. Figure $2 \mathrm{a}$ and $2 \mathrm{~b}$ are photos of $\mathrm{PVDF} /$ PCL $=90 / 10$ system before and after being etched, respectively. The pits in the fractured surface of etched 90/10 sample indicated that the PVDF and PCL showed the sea-island morphology. When the content of PCL increased, gullies appeared in the photo of etched 83/17 sample. It was clear that cocontinuous phase morphologies presented in the photo of etched 62/38 sample. By recalling the results of Figure 1, It suggested that the conductivity of composites became much better with the phase morphologies changing from sea-island to co-continuous. It meant that co-continuous phase enabled the formation of conductive pathways with MWNT net-

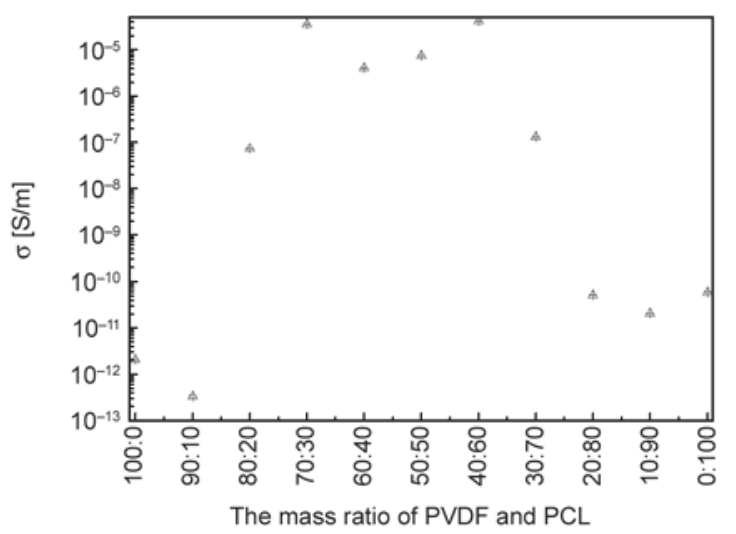

Figure 1. Conductive properties of $1.5 \mathrm{wt} \% \mathrm{MWNT} / \mathrm{PCL} /$ PVDF with different mass ratio of PVDF and PCL work. The sharp decrease after PVDF/PCL of 40/60 was attributed to decrease of the relative concentration of MWNT in PCL since MWNT content was fixed to $1.5 \mathrm{wt} \%$ of the whole component.

The distribution of MWNT is another important factor in this ternary system. The wetting parameter $\left(\omega_{\mathrm{a}}\right)$ can predict the selective distribution of MWNT in those immiscible polymer blends [29, 30]. For MWNT/PCL/PVDF composites, it can be described by Equation (1):

$\omega_{\mathrm{a}}=\frac{\gamma_{\mathrm{MWNT}-\mathrm{PVDF}}+\gamma_{\mathrm{MWNT}-\mathrm{PCL}}}{\gamma_{\mathrm{PVDF}-\mathrm{PCL}}}$

where $\gamma_{\text {MWNT-PVDF, }} \gamma_{\text {MWNT-PCL }}$ and $\gamma_{\text {PVDF-PCL }}$ represented the interfacial tensions between MWNT and PVDF matrix, between MWNT and PCL matrix, and between PVDF and PCL. If $\omega_{\mathrm{a}}>1$, MWNT will preferentially distribute in PCL. If $\omega_{\mathrm{a}}<-1$, MWNT will preferentially distribute in PVDF. And if $-1<\omega_{\mathrm{a}}<1$, MWNT are distributed in the interface between PVDF and PCL. The interfacial tension is calculated by the surface energies of the components including their dispersive and polar parts. According to the theory of $\mathrm{Wu}[31]$, the interfacial tensions between two phases $\left(\gamma_{12}\right)$ can be estimated by the harmonic-mean equation (Equation (2)) and geometric-mean equation ((Equation (3)):

Harmonic-mean equation

$\gamma_{12}=\gamma_{1}+\gamma_{2}-4\left(\frac{\gamma_{1 \mathrm{~d}} \gamma_{2 \mathrm{~d}}}{\gamma_{1 \mathrm{~d}}+\gamma_{2 \mathrm{~d}}}+\frac{\gamma_{1 \mathrm{p}} \gamma_{2 \mathrm{p}}}{\gamma_{1 \mathrm{p}}+\gamma_{2 \mathrm{p}}}\right)$

Geometric-mean equation

$\gamma_{12}=\gamma_{1}+\gamma_{2}-2\left(\sqrt{\gamma_{1 \mathrm{~d}} \gamma_{2 \mathrm{~d}}}+\sqrt{\gamma_{1 \mathrm{p}} \gamma_{2 \mathrm{p}}}\right)$

where $\gamma$ represents the surface tension and the subscripts 1 and 2 stand for phases 1 and 2, respectively. $\gamma_{d}$ is the dispersion component of surface tension, and $\gamma_{p}$ is the polar component. The relationship of $\gamma$, $\gamma_{\mathrm{d}}$ and $\gamma_{\mathrm{p}}$ can be expressed by the equation: $\gamma=$ $\gamma_{\mathrm{d}}+\gamma_{\mathrm{p}}$. The Geometric-mean equation is suitable to estimate the interfacial energy between low-energy materials and high-energy materials, such as mercury, silica, metallic oxide, etc. [31]. Compared to geometric-mean equation, the harmonic-mean equation is more suitable to the interfacial energy between low-energy materials, such as polymers, organic liquids, water, etc. [31]. Thus, harmonic-mean equa- 


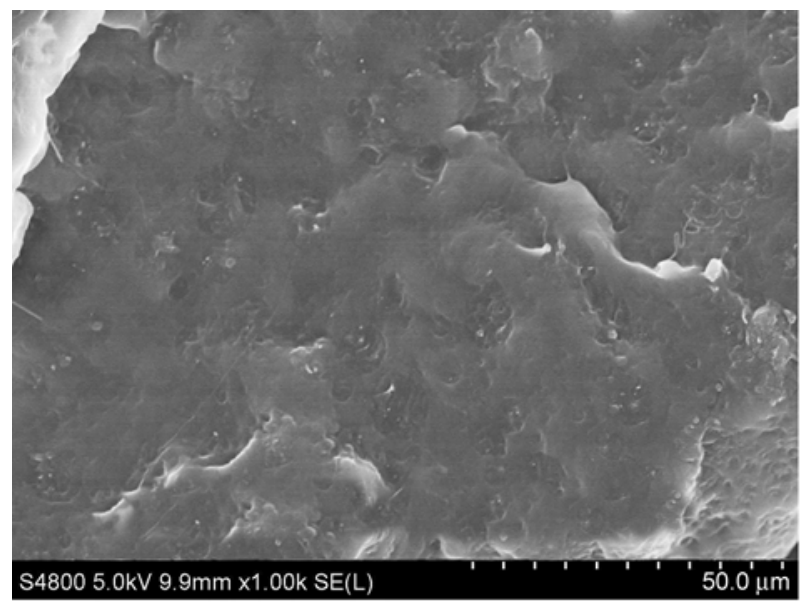

a) $\mathrm{PVDF} / \mathrm{PCL}=90: 10 \mathrm{wt} \%$

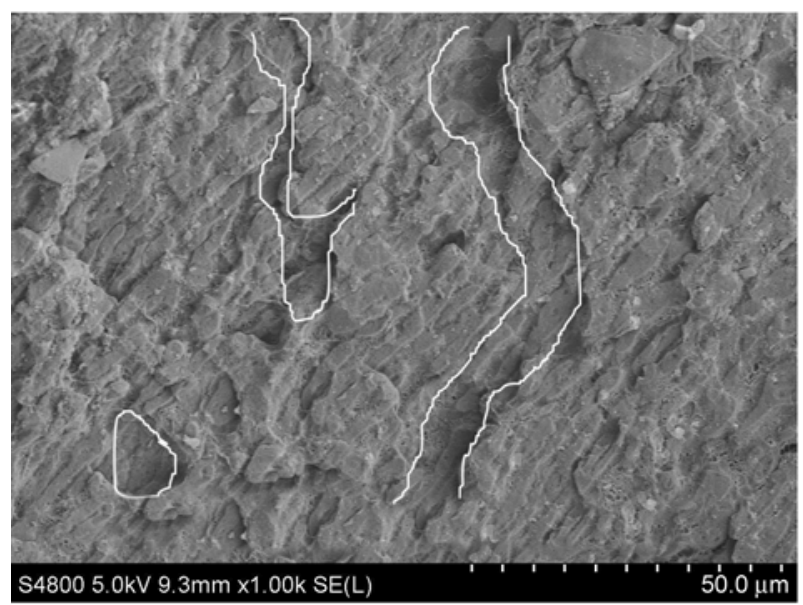

c) $\mathrm{PVDF} / \mathrm{PCL}=83: 17 \mathrm{wt} \%$

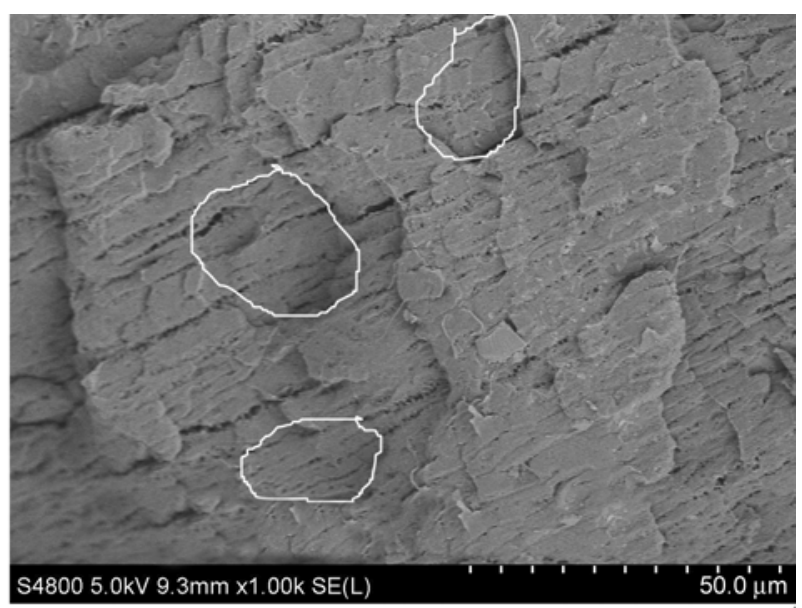

b) $\mathrm{PVDF} / \mathrm{PCL}=90: 10 \mathrm{wt} \%$

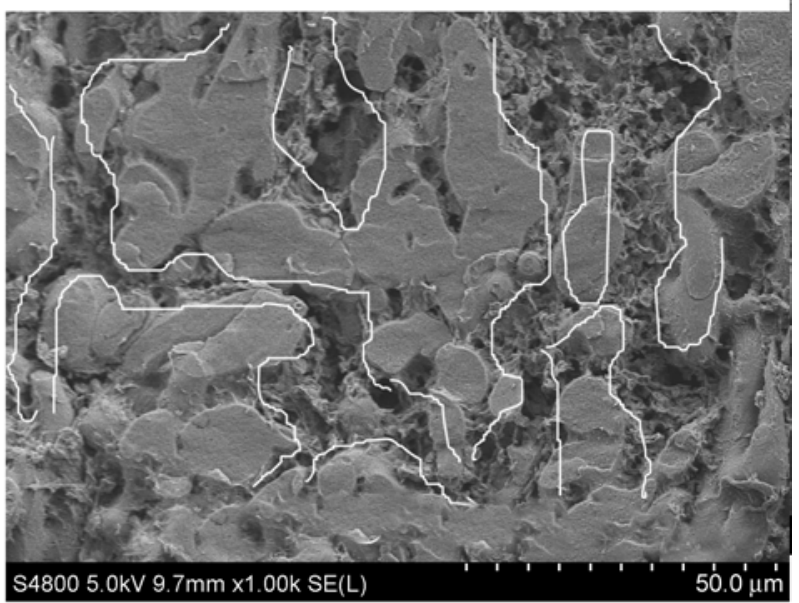

d) PVDF/PCL $=62: 38 \mathrm{wt} \%$

Figure 2. Morphology of the $1.5 \mathrm{wt} \% \mathrm{MWNT} / \mathrm{PCL} / \mathrm{PVDF}$ with different concentration of PVDF: (a) PVDF/PCL = 90/10, (b) $\mathrm{PVDF} / \mathrm{PCL}=90 / 10$ (after being etched), (c) PVDF/PCL $=83 / 17$ (after being etched), (c) PVDF/PCL $=62 / 38$ (after being etched)

tion is utilized here to evaluate the interfacial energy between MWNT and polymers.

In order to understand the interfacial effect of polymer melts during processing, the interfacial ten-

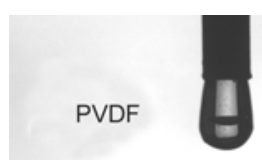

a)

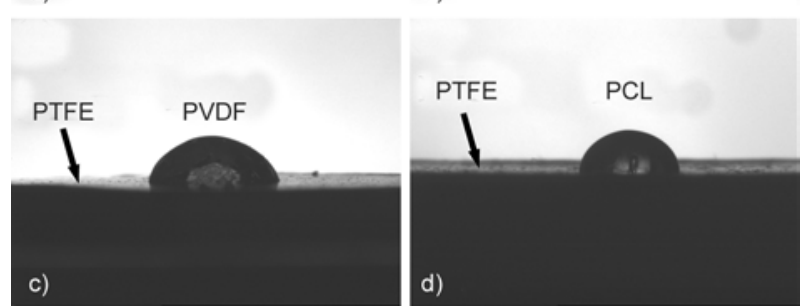

Figure 3. The drops of PVDF and PCL at $200^{\circ} \mathrm{C}$ : (a) and (b) pendant drops, (c) and (d) sessile drops on PTFE sions of the polymer melts at processing temperature need to be tested. The pendant drop method is the effective method to measure the interfacial tensions of polymer melts [27, 28]. Images of pendant drops of PVDF and PCL melt were shown in Figure 3. Basing on the Young-Laplace theory, the interfacial tensions can be calculated by the size parameters. The results are calculated by fitting and listed in Table 1.The $\gamma_{d}$ and $\gamma_{p}$ cannot be obtained directly by this method and it can be measured by a known and stable material at testing temperature. Nonpolar PTFE was chosen as the base plate to measure the $\gamma d$ of PVDF and PCL. The $\gamma d$ of PVDF and PCL at $200^{\circ} \mathrm{C}$ is calculated by Equation (4):

$$
\sigma^{\mathrm{d}}=\frac{\sigma^{2}}{4 \sigma_{\mathrm{PTFE}}}(1+\cos \theta)^{2}
$$

where $\sigma^{\mathrm{d}}$ represents the dispersion component of surface tension of the polymer melt, $\sigma$ is the surface tension of the polymer melt, $\sigma_{\mathrm{PTFE}}$ is the surface 
Table 1. Surface tensions results of PVDF and PCL at $200^{\circ} \mathrm{C}$ using the pendant drops method

\begin{tabular}{|l|c|c|c|}
\hline & $\begin{array}{c}\boldsymbol{\gamma} \\
{\left[\mathbf{m J} \cdot \mathbf{m}^{-2}\right]}\end{array}$ & $\begin{array}{c}\gamma_{\mathbf{d}} \\
{\left[\mathbf{m J} \cdot \mathbf{m}^{-2}\right]}\end{array}$ & $\begin{array}{c}\gamma_{\mathbf{p}} \\
{\left[\mathbf{m J} \cdot \mathbf{m}^{-2}\right]}\end{array}$ \\
\hline PCL & 29.81 & 21.10 & 8.71 \\
\hline PVDF & 29.16 & 26.11 & 3.05 \\
\hline
\end{tabular}

tension of the PTFE base plate. $\gamma_{\mathrm{p}}$ can be calculated by the equation: $\gamma_{\mathrm{p}}=\gamma-\gamma_{\mathrm{d}}$.

The surface tension of MWNT is obtained from related references $[23,25]$. The $\gamma, \gamma_{\mathrm{d}}, \gamma_{\mathrm{p}}$ values of MWNT are $45.3,18.4,26.9 \mathrm{~mJ} \cdot \mathrm{m}^{-2}$ respectively. Then the interfacial energies between MWNT and polymers at $200^{\circ} \mathrm{C}$ were calculated by using the harmonic-mean equation: $\gamma_{\text {MWNT-PVDF }}=20.33 \mathrm{~mJ} \cdot \mathrm{m}^{-2}$, $\gamma_{\text {MWNT-PCL }}=9.48 \mathrm{~mJ} \cdot \mathrm{m}^{-2}$ and $\gamma_{\text {PVDF-PCL }}=3.26 \mathrm{~mJ} \cdot \mathrm{m}^{-2}$. From Equation (1), $\omega_{\mathrm{a}}$ value of 3.33 is obtained. Because of $\omega_{\mathrm{a}}>1$, these calculations provide an explanation for the selective distribution of MWNT in the PCL phase by interfacial effects when thermodynamic equilibrium is reached.

To further observe the dispersion of MWNT in composites, TEM micrographs of $1.5 \mathrm{wt} \% \mathrm{MWNT} / \mathrm{PCL} /$ PVDF were shown in Figure 4. PVDF and PCL phase cannot be distinguished by the TEM images all alone, but the dispersion of WMTNT in MWNT/PCL/ PVDF composite is easy to be seen in TEM images. The dispersion of MWNT could indicate the distribution of PCL phase since studies on interfacial effect confirmed the selective localization of MWNT in PCL. It can be seen that MWNT formed large aggregates in the $\mathrm{PVDF} / \mathrm{PCL}=90 / 10$ system (Figure 4a), which demonstrated sea-island morphology of the composites. With the increase of PCL content, the phase morphology of PVDF/PCL began to change from sea-island to the co-continuous as dis-

persion of MWNT was improved (Figure 4b). Welldeveloped co-continuous morphologies are clearly visible in 62/38 system, MWNT selectively filled the zonal PCL phase and formed a network (Figure 4c). As the result, co-continuous blend phase (first percolation) in combination with the formation of MWNT networks by selectively filling the blend phase (second percolation) built the desired structure of double percolation in MWNT/PVDF/PCL composites.

Conductive properties of different proportions of PVDF and PCL with different concentration of MWNT were displayed in Figure 5.There was no percolation threshold below the MWNT concentration of $2.5 \mathrm{wt} \%$ in MWNT/PVDF composites, similar to the reported studies on MWNT/PVDF prepared by melt blending method $[9,10,32]$. The percolation threshold of MWNT/PCL is about $1.5 \mathrm{wt} \%$ owing to better compatibility between PCL and MWNT. The conductivity of MWNT/PVDF/PCL =

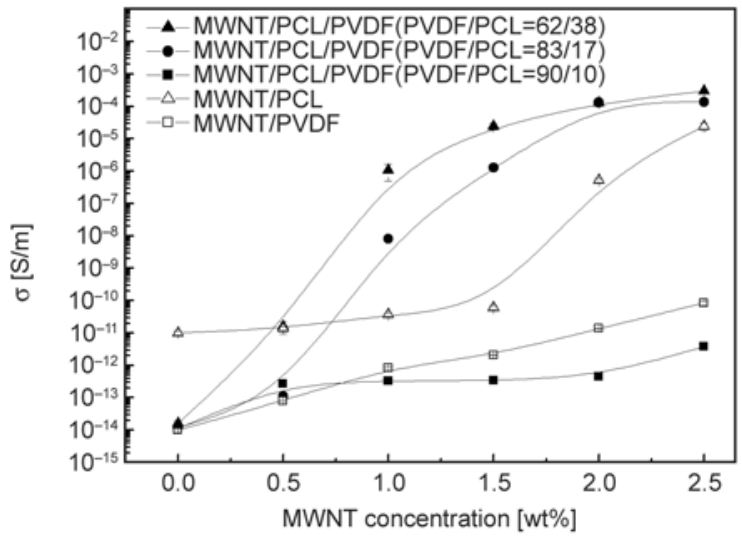

Figure 5. Conductive properties of different proportions of PVDF and PCL matrixes with different concentration of MWNT through melt blending

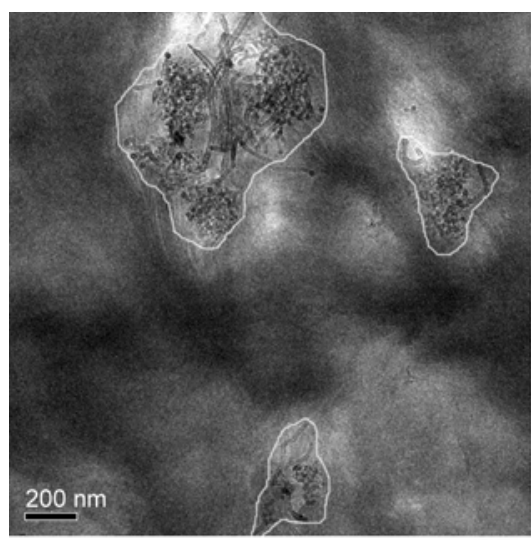

a)

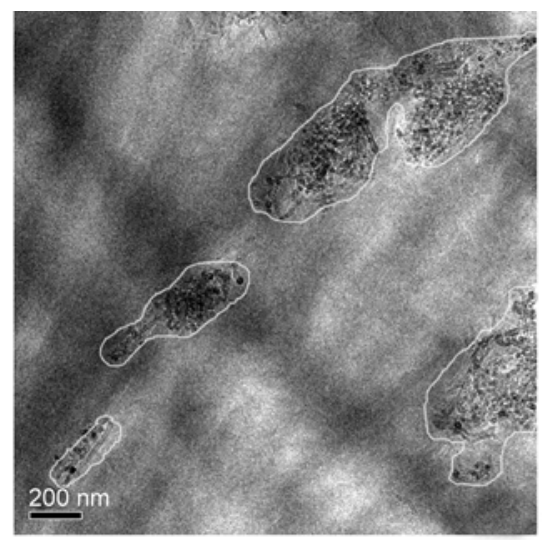

b)

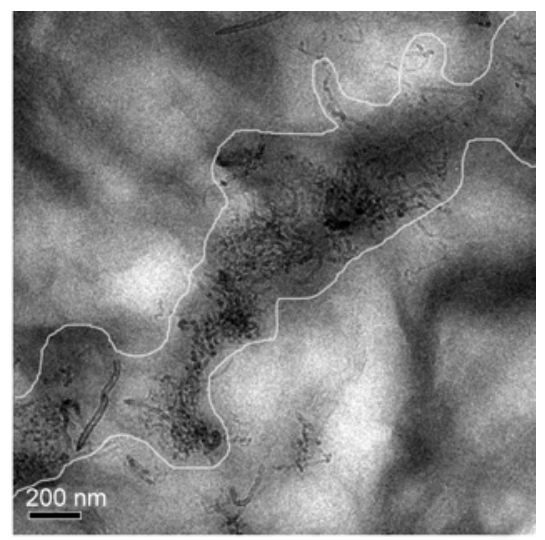

c)

Figure 4. TEM micrographs of $1.5 \mathrm{wt} \% \mathrm{MWNT} / \mathrm{PCL} / \mathrm{PVDF}$ : (a) $\mathrm{PVDF} / \mathrm{PCL}=90 / 10$, (b) $\mathrm{PVDF} / \mathrm{PCL}=83 / 17$, (c) $\mathrm{PVDF} / \mathrm{PCL}=62 / 38$ 
90/10 system was poorer than that of MWNT/ PVDF because MWNT concentrated in island-phase of PCL and conductive route could not be formed, as demonstrated by TEM observation. The conductivity of MWNT/PVDF/PCL $=83 / 17$ and MWNT/ $\mathrm{PVDF} / \mathrm{PCL}=62 / 38$ system showed the significantly lower electrical percolation threshold $(<0.5 \mathrm{wt} \%)$. Comparing conductivity of composites with cocontinuous phase to those of MWNT/PCL, MWNT/ PVDF and MWNT/PVDF/PCL $=90 / 10$ nanocomposites, the improvement of conductivity over some decades at the same filler loading can be clearly seen. These gave another hint to double percolation structure in co-continuous blend phase

\section{Conclusions}

In conclusion, a strategy of designing ternary system and selective localization of MWNT in co-continuous blend phase were taken to prepare MWNT/ PVDF conductive material with low percolation threshold by melt blending. Investigation on the interfacial tensions of PVDF and PCL melts at mixing temperature and calculations of wetting coefficient confirmed selectively localization of MWNT in PCL phase. The formation of well-developed double percolated morphologies by adjusting the proportion of blends was observed. PVDF nanocomposites with low percolation threshold $(<0.5 \mathrm{wt} \%)$ was acquired. Preferential localization of conductive filler in co-continuous blends opens perspectives for the development of conductive PVDF based material.

\section{Acknowledgements}

The authors are grateful for the support of the Natural Science Foundation of China (Grant: 51173207), the Science and Technology Department of Guangdong Province (Grants: 2011B090500004, 2012B091100313, 2012A090100006 and 2013C2FC0009), and Key projects of Guangdong Education Office (Grant: cxzd1101).

\section{References}

[1] Wang J., Wu J., Xu W., Zhang Q., Fu Q.: Preparation of poly(vinylidene fluoride) films with excellent electric property, improved dielectric property and dominant polar crystalline forms by adding a quaternary phosphorus salt functionalized graphene. Composites Science and Technology, 91, 1-7 (2014). DOI: $10.1016 /$ j.compscitech.2013.11.002
[2] Ting Y., Gunawan H., Sugondo A., Chiu C-W.: A new approach of polyvinylidene fluoride (PVDF) poling method for higher electric response. Ferroelectrics, 446, 28-38 (2013). DOI: $10.1080 / 00150193.2013 .820983$

[3] Liu Z. H., Pan C. T., Lin L. W., Li H. W., Ke C. A., Huang J. C., Wang P. S.: Mechanical properties of piezoelectric PVDF/MWCNT fibers prepared by flat/ hollow cylindrical near-field electrospinning process. in '2013 $8^{\text {th }}$ IEEE International Conference on Nano/ Micro Engineered and Molecular Systems (NEMS), Suzhou, China' 707-710 (2013).

DOI: 10.1109/NEMS.2013.6559827

[4] Wang L., Dang Z-M.: Carbon nanotube composites with high dielectric constant at low percolation threshold. Applied Physics Letters, 87, 042903/1-042903/3 (2005).

DOI: $10.1063 / 1.1996842$

[5] Hu B., Li D., Manandharm P., Fan Q., Kasilingam D., Calvert P.: CNT/conducting polymer composite conductors impart high flexibility to textile electroluminescent devices. Journal of Materials Chemistry, 22, 1598-1605 (2012).

DOI: $10.1039 / \mathrm{C} 1 \mathrm{JM} 14121 \mathrm{~J}$

[6] Thomas J. A., Iutzi R. M., McGaughey A. J. H.: Thermal conductivity and phonon transport in empty and water-filled carbon nanotubes. Physical Review B, 81, 045413/1-045413/7 (2010).

DOI: $10.1103 /$ PhysRevB.81.045413

[7] Bui K., Grady B. P., Papavassiliou D. V.: Heat transfer in high volume fraction CNT nanocomposites: Effects of inter-nanotube thermal resistance. Chemical Physics Letters, 508, 248-251 (2011). DOI: 10.1016/j.cplett.2011.04.005

[8] Ke K., Wang Y., Zhang K., Luo Y., Yang W., Xie B-H., Yang M-B.: Melt viscoelasticity, electrical conductivity, and crystallization of PVDF/MWCNT composites: Effect of the dispersion of MWCNTs. Journal of Applied Polymer Science, 125, 49-57 (2012).

DOI: 10.1002/app.36293

[9] Kim G. H., Hong S. M.: Structures and physical properties of carbon nanotube reinforced PVDF composites. Molecular Crystals and Liquid Crystals, 472, 161169 (2007). DOI: $10.1080 / 15421400701545494$

[10] Hong S. M., Nam Y. W., Hwang S. S., Chae D. W.: Physical properties of multi-walled carbon nanotubefilled PVDF composites prepared by melt compounding. Molecular Crystals and Liquid Crystals, 464, 195203 (2007).

DOI: $10.1080 / 15421400601030969$

[11] Yang D-D., Xu H-P., Wang J-R., Wu Y-H.: Nickel-multiwalled carbon nanotubes/polyvinylidene fluoride composites with high dielectric permittivity. Journal of Applied Polymer Science, 130, 3746-3752 (2013). DOI: 10.1002/app.39645 
[12] Yang C., Lin Y., Nan C. W.: Modified carbon nanotube composites with high dielectric constant, low dielectric loss and large energy density. Carbon, 47, 1096-1101 (2009). DOI: $10.1016 /$ j.carbon.2008.12.037

[13] Li Q., Xue Q., Zheng Q., Hao L., Gao X.: Large dielectric constant of the chemically purified carbon nanotube/ polymer composites. Materials Letters, 62, 4229-4231 (2008).

DOI: $10.1016 /$ j.matlet.2008.06.047

[14] Dang Z-M., Wang L., Yin Y., Zhang Q., Lei Q-Q.: Giant dielectric permittivities in functionalized carbon-nanotube/electroactive-polymer nanocomposites. Advanced Materials, 19, 852-857 (2007).

DOI: $10.1002 / \mathrm{adma} .200600703$

[15] Liu L., Wang Y., Xiang F., Li Y., Han L., Zhou Z.: Effects of functionalized multiwalled carbon nanotubes on the morphologies and mechanical properties of PP/EVA blend. Journal of Polymer Science Part B: Polymer Physics, 47, 1481-1491 (2009).

DOI: $10.1002 /$ polb.21749

[16] Göldel A., Kasaliwal G., Pötschke P.: Selective localization and migration of multiwalled carbon nanotubes in blends of polycarbonate and poly(styrene-acrylonitrile). Macromolecular Rapid Communications, 30, 423-429 (2009).

DOI: $10.1002 /$ marc.200800549

[17] Pang H., Yan D-X., Bao Y., Chen J-B., Chen C., Li Z-M.: Super-tough conducting carbon nanotube/ultrahighmolecular-weight polyethylene composites with segregated and double-percolated structure. Journal of Materials Chemistry, 22, 23568-23575 (2012).

DOI: $10.1039 / \mathrm{C} 2 \mathrm{JM} 34793 \mathrm{H}$

[18] Jin S-H., Lee D-S.: Electrical and rheological properties of double percolated poly(methyl methacrylate)/ multiwalled carbon nanotube nanocomposites. Journal of Nanoscience and Nanotechnology, 7, 3847-3851 (2007). DOI: 10.1166/jnn.2007.058

[19] Bose S., Bhattacharyya A. R., Bondre A. P., Kulkarni A. R., Pötschke P.: Rheology, electrical conductivity, and the phase behavior of cocontinuous PA6/ABS blends with MWNT: Correlating the aspect ratio of MWNT with the percolation threshold. Journal of Polymer Science Part B: Polymer Physics, 46, 1619-1631 (2008). DOI: $10.1002 /$ polb.21501

[20] Li Y., Shimizu H.: Conductive PVDF/PA6/CNTs nanocomposites fabricated by dual formation of cocontinuous and nanodispersion structures. Macromolecules, 41, 5339-5344 (2008). DOI: $10.1021 / \mathrm{ma} 8006834$

[21] Sumita M., Sakata K., Asai S., Miyasaka K., Nakagawa H.: Dispersion of fillers and the electrical conductivity of polymer blends filled with carbon black. Polymer Bulletin, 25, 265-271 (1991).

DOI: $10.1007 / \mathrm{BF} 00310802$
[22] Baudouin A-C., Devaux J., Bailly C.: Localization of carbon nanotubes at the interface in blends of polyamide and ethylene-acrylate copolymer. Polymer, 51, 1341-1354 (2010). DOI: 10.1016/j.polymer.2010.01.050

[23] Baudouin A-C., Bailly C., Devaux J.: Interface localization of carbon nanotubes in blends of two copolymers. Polymer Degradation and Stability, 95, 389-398 (2010). DOI: $10.1016 /$ j.polymdegradstab.2009.11.007

[24] Goldel A., Marmur A., Kasaliwal G. R., Potschke P., Heinrich G.: Shape-dependent localization of carbon nanotubes and carbon black in an immiscible polymer blend during melt mixing. Macromolecules, 44, 60946102 (2011). DOI: $10.1021 / \mathrm{ma} 200793 \mathrm{a}$

[25] Wu D., Zhang Y., Zhang M., Yu W.: Selective localization of multiwalled carbon nanotubes in poly(E-caprolactone)/polylactide blend. Biomacromolecules, 10, 417-424 (2009).

DOI: $10.1021 / \mathrm{bm} 801183 \mathrm{f}$

[26] Göldel A., Kasaliwal G., Pötschke P.: Selective localization and migration of multiwalled carbon nanotubes in blends of polycarbonate and poly(styrene-acrylonitrile). Macromolecular Rapid Communications, 30, 423-429 (2009).

DOI: $10.1002 /$ marc. 200800549

[27] Arashiro E. Y., Demarquette N. R.: Use of the pendant drop method to measure interfacial tension between molten polymers. Materials Research, 2, 23-32 (1999). DOI: $10.1590 / \mathrm{S} 1516-14391999000100005$

[28] Stauffer C. E.: The measurement of surface tension by the pendant drop technique. The Journal of Physical Chemistry, 69, 1933-1938 (1964). DOI: $10.1021 / \mathrm{j} 100890 \mathrm{a} 024$

[29] Göldel A., Kasaliwal G., Pötschke P.: Selective localization and migration of multiwalled carbon nanotubes in blends of polycarbonate and poly(styrene-acrylonitrile). Macromolecular Rapid Communications, 30, 423-429 (2009).

DOI: $10.1002 /$ marc.200800549

[30] Baudouin A-C., Devaux J., Bailly C.: Localization of carbon nanotubes at the interface in blends of polyamide and ethylene-acrylate copolymer. Polymer, 51, 1341-1354 (2010). DOI: 10.1016/j.polymer.2010.01.050

[31] Wu S.: Polymer interface and adhesion. Marcel Dekker New York (1982).

[32] Ke K., Wang Y., Zhang K., Luo Y., Yang W., Xie B-H., Yang M-B.: Melt viscoelasticity, electrical conductivity, and crystallization of PVDF/MWCNT composites: Effect of the dispersion of MWCNTs. Journal of Applied Polymer Science, 125, 49-57 (2012). DOI: $10.1002 /$ app.36293 